Artículo Original

\title{
Las especies del grupo de Hypodynerus caupolicanus (Reed, 1893) (Hymenoptera: Vespidae: Eumeninae), con descripción de dos nuevas especies de Chile
}

Species of the Hypodynerus caupolicanus (Reed, 1893) group (Hymenoptera: Vespidae:

Eumeninae), with description of two new species from Chile

\author{
Roberto Barrera-Medina ${ }^{\mathbb{R}}$ y Bolívar R. Garcete-Barrett ${ }^{2,3}(\mathbb{C}$
}

${ }^{1}$ Pasaje 2 casa 279, Villa Empart Puente Alto, Santiago, Chile. Nacional de Historia Natural del Paraguay, Km 10 1/2, Sucursal 1 Campus U.N.A., 2169 CDP, Central XI, San Lorenzo, Paraguay. ${ }^{3}$ Departamento de Biología clo Dirección de Investigación, Facultad de Ciencias Exactas y Naturales, Universidad Nacional de Asunción, Casilla de Correo 1039, Campus U.N.A., 2160 CDP, Central XI, San Lorenzo, Paraguay.

ZooBank: urn:lsid:zoobank.org:pub:D40324C6-9EE4-485C-B959-73E1823F142A

https: / / doi.org/10.35249/ rche.47.1.21.16

Resumen. Se describen e ilustran dos nuevas especies del grupo de Hypodynerus caupolicanus, provenientes de la zona central de Chile. Se actualiza la distribución geográfica de todas las especies del grupo, se presentan esquemas de coloración para cada una de ellas y se entrega una clave para su identificación.

Palabras clave: Avispas alfareras; Chile central; clave; esquema de coloración.

Abstract. Two new species of the Hypodynerus caupolicanus group from central Chile are described and illustrated. The geographical distribution of all species in the group is updated, coloring schemes are presented for each one of them species and a key is provided for their identification.

Key words: Central Chile; coloring schemes; key; pottery wasps.

\section{Introducción}

El género Hypodynerus Saussure, 1855 está representado en Sudamérica por alrededor de 50 especies incluidas hasta el momento en seis grupos afines distribuidos a lo largo de la cordillera de los Andes (Barrera-Medina 2011). En Chile este taxón está conformado por 29 especies presentes entre las regiones de Arica y Parinacota y Magallanes (BarreraMedina y Garcete-Barrett 2016). La última lista de especies reportadas para Chile fue proporcionada por Barrera-Medina (2011), posteriormente, Barrera-Medina y GarceteBarrett (2016) reportan la presencia de Hypodynerus rufinodis (du Buysson, 1912) en el extremo norte del país.

Willink (1970) definió los límites de los seis grupos reconocidos para este género y revisó el grupo de Hypodynerus humeralis (Haliday, 1836); el mismo autor (Willink 1978a, 1978b) revisó los grupos de $H$. tuberculiventris (Spinola, 1851) y H. excipiendus (Spinola,

Recibido 31 Agosto 2020 / Aceptado 22 Marzo 2021 / Publicado online 31 Marzo 2021

Editor Responsable: José Mondaca E. 
1851), quedando pendiente el estudio de los grupos de H. arechavaletae (Brèthes, 1903), H. caupolicanus (Reed, 1893), H. chiliotus (de Saussure, 1851) y H. labiatus (Haliday, 1836). Con respecto al grupo de H. tuberculiventris, Willink (1970) le asignó como especie tipo a H. ruficollis (Spinola, 1851), y posteriormente a H. tuberculiventris (Willink 1978b).

De acuerdo con lo indicado por Willink (1970), el grupo de H. caupolicanus corresponde a avispas de tamaño medio que presentan el pronoto con reborde o lamela anterosuperior continua, o apenas interrumpida medialmente; segundo esternito abdominal fuertemente angulado en la base, sin protuberancia mediana; ángulos posterolaterales del propodeo redondeados o con una carena poco definida. Barrera-Medina (2011) señala que el grupo de $H$. caupolicanus está formado por $H$. antuco (de Saussure, 1851), H. bruchii (Brèthes, 1903), H. caupolicanus, H. cerberus Bequaert y Ruiz, 1943 y H. herbsti Bequaert y Ruiz, 1943. Estos dos últimos autores describen a $H$. herbsti y $H$. cerberus como subespecies de H. caupolicanus, basados en las diferencias de coloración y tamaño entre estas especies, mientras que Willink y Chiappa (1993) las elevan a la categoría de especies sin explicar las razones del cambio propuesto.

Los objetivos de este trabajo son actualizar el conocimiento de las especies del grupo de $H$. caupolicanus, describir dos nuevas especies de Chile, y establecer la nueva ubicación de H. antuco en el grupo de H. labiatus propuesto por Willink (1970). Adicionalmente se ilustran todas las especies del grupo hasta ahora conocidas y se entrega una nueva clave que facilite la identificación.

\section{Materiales y Métodos}

Se revisaron 83 especímenes del grupo $H$. caupolicanus pertenecientes a la colección de insectos del Museo Nacional de Historia Natural de Chile (MNHN) y la colección privada del primer autor (CRBM). Para las observaciones y el estudio de los especímenes se utilizó un estereomicroscopio con aumentos de 20x y 40x. Las fotografías fueron tomadas con una cámara réflex Canon EOS T5 Rebel de 20 megapíxeles y un tubo de extensión de 3 partes; para las mediciones se utilizó un ocular con reglilla graduada y un pie de metro digital. La medida del largo de las alas se consideró desde las tégulas (no incluyéndolas) hasta el borde apical; las medidas del mesosoma se tomaron a nivel de las paratégulas, y las del metasoma al nivel del ancho máximo del segundo tergo; todas las medidas presentadas corresponden al promedio $(\bar{X})$ de las mediciones realizadas a los ejemplares.

\section{Resultados}

La identificación del material hasta el nivel género se realizó siguiendo a Carpenter y Garcete-Barrett (2003). El trabajo de Willink (1970) permitió ubicar a 60 en ejemplares estudiados en el grupo de $H$. caupolicanus y 23 en el grupo de H. labiatus, tras el uso de las claves de Reed (1893), Brèthes (1903), Zavattari (1912), Bequaert y Ruiz (1943) y la comparación morfológica con especies próximas se concluyó que 23 ejemplares correspondieron a H. antuco, cinco a H. bruchii, cinco a H. caupolicanus, dos a H. cerberus y 13 a $H$. herbsti. Adicionalmente 35 ejemplares correspondieron a dos diferentes especies desconocidas para la ciencia que son descritas a continuación.

\section{Hypodynerus aceitunoi Barrera-Medina y Garcete-Barrett, sp. nov.}

(Figs. 1A-1E, 3A)

Localidad tipo. Cuesta Lo Prado, Provincia de Santiago, Región Metropolitana de Santiago, Chile. 
Diagnosis. Avispa de tamaño mediano (alas anteriores 9,1-11,4 mm; ancho máximo de la cabeza $\bar{X}: 3,6 \mathrm{~mm}$, ancho máximo del mesosoma $\bar{X}: 3,9 \mathrm{~mm}$; ancho máximo del metasoma $\overline{\mathrm{X}}: 3,5 \mathrm{~mm}$ ). Tegumento negro con los siguientes diseños marfileños: banda apical desarrollada en el pronoto, mancha dorsal en las mesopleuras, banda en el postescudete, bandas apicales en el primer y segundo tergo, los diseños marfileños pueden no estar presentes o disminuidos; clípeo mayormente ferrugíneo con bordes negros, en algunos casos formando una amplia mancha central ferrugínea en las hembras, convexo en vista lateral, antenas y tégulas ferrugíneas, con excepción de los cuatro últimos esternitos que son negros en su parte externa, pronoto con reborde anterosuperior continuo, ángulos posterolaterales del propódeo redondeados y segundo esterno del metasoma sin protuberancias.

Descripción. Holotipo hembra. Coloración (Fig. 3A): negra con las siguientes partes marfil: línea angosta superior en la órbita externa; banda apical desarrollada en el pronoto; mancha dorsal en las mesopleuras; postescudete; bandas apicales en primer y segundo tergo. Son ferrugíneas: pequeña mancha en la frente entre la base de las antenas con forma de $\mathrm{V}$ invertida en algunos casos dividida medialmente, presente en el $38 \%$ de los ejemplares revisados, en el resto ausente, antenas, con excepción de la parte externa de los últimos cuatro antenitos que es negra; clípeo mayormente ferrugíneo con bordes negros, en algunos casos formado una amplia mancha central ferrugínea; mandíbulas en sus bordes externo, interno y en su ápice ferrugíneo oscuro; tégulas; trocánteres; patas a partir de menos de la mitad de los fémures. Alas en gran parte ferrugíneas, sólo con el ápice en parte infumado, algunos de los diseños marfileños pueden no estar presentes en ejemplares ( $18 \%$ de los ejemplares revisados no presentan mancha dorsal en las mesopleuras y postescudete marfileño). Cabeza (Figs. 1C-1D): más ancha que larga (ancho $\bar{X}: 3,6 \mathrm{~mm}$; largo $\overline{\mathrm{X}}: 3,2 \mathrm{~mm}$ ); distancia interorbital máxima mayor que el largo del ojo $(1: 0,7)$; órbitas internas profundamente escotadas en la mitad superior, de modo que la distancia entre las escotaduras es casi el doble de la distancia interorbital media (1:0,6); clípeo convexo en vista lateral, más ancho que largo (1:0,9), apicalmente emarginado, sin reborde translúcido, y con dos dientes apicales redondeados y levemente proyectados lateralmente; mandíbulas largas con 4 dientes (considerando como un diente el ápice de la mandíbula), cara dorsal provista de carenas longitudinales y cara ventral cóncava; antenas: escapo más de tres veces más largo que ancho (1:0,2). Mesosoma (Figs. 1A-1B): con apariencia lustrosa; más largo que ancho (1:0,7); pronoto con reborde anterosuperior continuo; propódeo con los ángulos posterolaterales redondeados, no carenados, y la superficie lateral en gran parte brillante, con rugosidades. Metasoma (Figs. 1A-1B): ancho máximo $\bar{X}: 3,6 \mathrm{~mm}$; en el primer tergo, la cara anterior pasa a la dorsal formando un ángulo fuerte con un surco longitudinal medio que da origen a una hendidura basal; el segundo esterno es redondeado basalmente en vista lateral. Puntuación: cabeza con puntos marcados, en el vertex de menor diámetro que el espacio entre ellos, hacia el clípeo y la escotadura de los ojos tiene un diámetro mayor que la distancia entre ellos; mesosoma con puntos marcados, mayoritariamente con un diámetro menor que el espacio entre ellos; clípeo con algunos puntos de un diámetro menor que el espacio entre ellos, en el ápice más marcados y de mayor tamaño que en su zona media y basal; primer tergo del metasoma con puntuación marcada que presenta un diámetro menor que el espacios entre ellos; en los demás tergos no se aprecia puntuación. Pilosidad: larga espaciada entre ella, negra sobre las zonas de tegumento negro y marfileña en las zonas de tegumento de ese color.

Macho. Desconocido. 


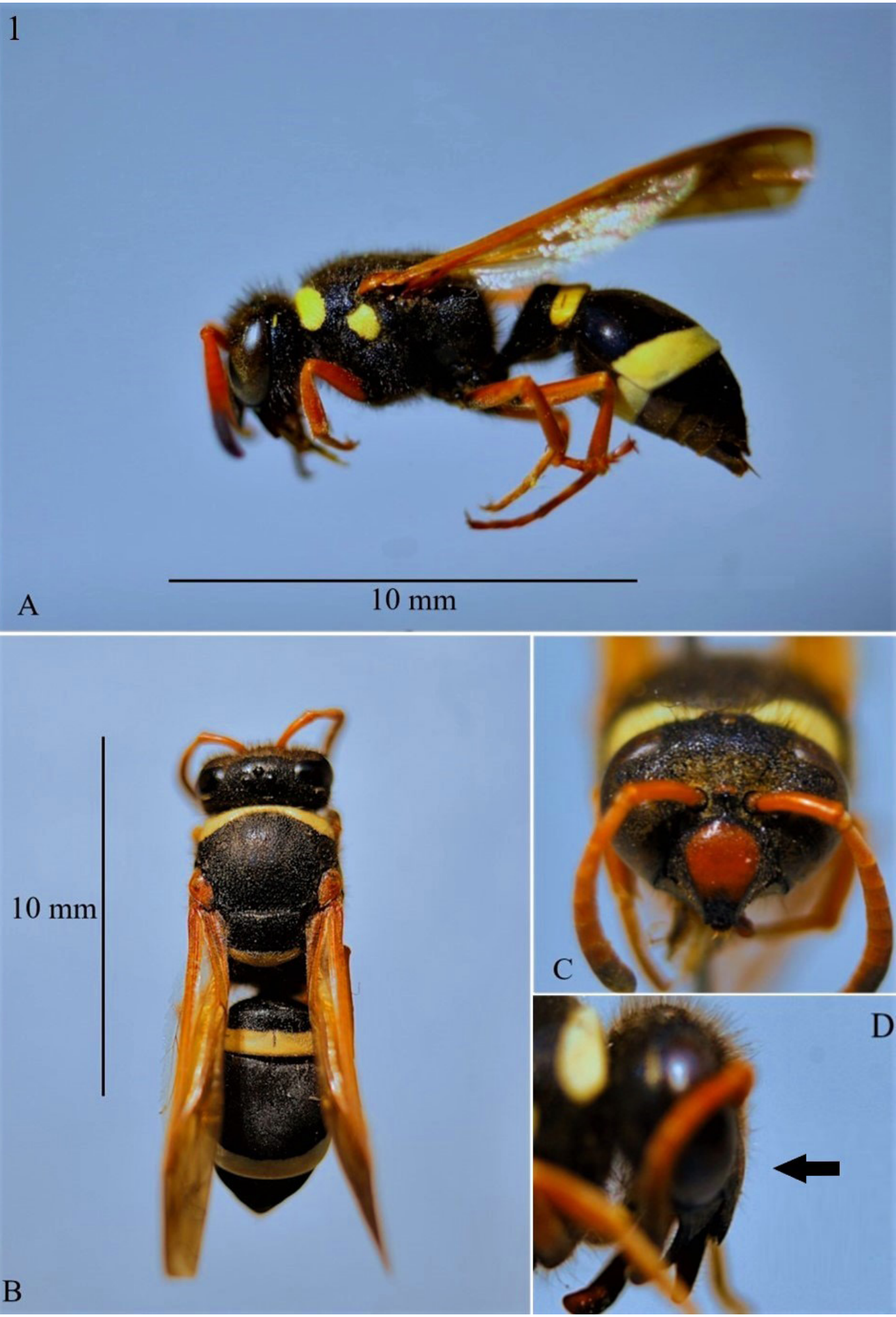

Figura 1. Hypodynerus aceitunoi sp. nov. A. Vista lateral izquierda. B. Vista dorsal. C. Cabeza vista frontal. D. Clípeo vista lateral. / A. Left side view. B. Dorsal view. C. Head frontal view. D. Clypeus lateral view. 
Material examinado. Holotipo hembra de: Chile, Región Metropolitana de Santiago,

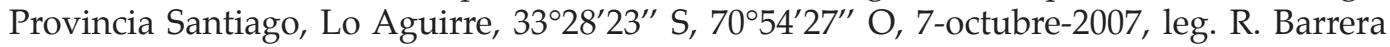
M. (MNHN). Paratipos: Chile, Región Metropolitana de Santiago, Provincia Santiago,

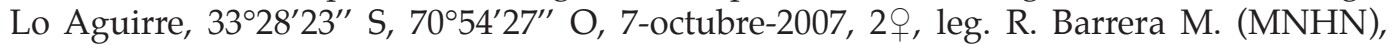
1-noviembre-2008, 5ㅇ, leg. R. Barrera M. (CRBM), cuesta Lo Prado, 12-octube-1998, 1 , leg. G. Aceituno (MNHN); Región de Coquimbo, Provincia de La Serena, Punta de Choros, 28-octubre-2009, 1, leg. C. Fortino(CRBM); Provincia del Elqui, Vicuña, 11-noviembre-2009, 6이 leg. G. Castillo (MNHN), 22-noviembre-1989, 1일. leg. L. Peña (CRBM), El Pangue, 16-20-octubre-1991, 1ㅇ, leg. L. Peña (MNHN); Provincia de Limarí, caleta El Sauce, 18-octubre-2009, 6일 leg. R. Barrera M. (MNHN); Región de Atacama, Provincia Huasco, Los Toyos, 8 al 11-octubre-2004, 2 , leg. R. Barrera M. (CRBM), 7 al 12-octube-2005, 1q, leg. R. Barrera M. (MNHN); Región de Atacama, Provincia de Huasco, Carrizal Bajo, 12-octubre 2011, 1 , leg. R. Barrera M. (CRBM), Chañaral de Aceituno, 9 al 11-octubre-2004, 1q, leg. R. Barrera M. (MNHN), camino a Llanos de Challe, 18-octubre-2008, 1ㅇ, leg. R. Barrera M. (MNHN), Llanos de Challe, 12-octubre-2011, 1q, leg. R. Barrera M. (MNHN).

Etimología. Especie dedicada a Guillermo Aceituno, entomólogo que ha realizado interesantes aportes a la entomofauna chilena y que nos proporcionó el primer ejemplar conocido de esta nueva especie.

Distribución. Chile, entre las regiones de Atacama y Metropolitana de Santiago, tramo que corresponde a las provincias biogeográficas de Atacama y Santiago (Morrone 2015).

\section{Hypodynerus ficheti Barrera-Medina y Garcete-Barrett, sp. nov.}

(Figs. 2A-2D, 3H)

Localidad tipo. Cordillera de Copiapó, Provincia Copiapó, Región de Atacama, Chile.

Diagnosis. Avispa de tamaño mediano, robusta (alas anteriores 10,3-11,3 mm; ancho máximo de la cabeza $\bar{X}: 3,9 \mathrm{~mm}$; ancho máximo del mesosoma $\overline{\mathrm{X}}: 4,8 \mathrm{~mm}$, ancho máximo del metasoma $\bar{X}: 4,6 \mathrm{~mm}$ ), con el patrón de coloración característico del género, negro con bandas marfil; clípeo ferrugíneo, con bordes laterales negros en las hembras, basalmente proyectado hacia adelante en vista lateral, apicalmente emarginado y con dos dientes apicales bien desarrollados y levemente proyectados lateralmente, banda marfil apical presente en el pronoto, tégulas ferrugíneas, metasoma con bandas marfil apicales en primer y segundo tergo. Pronoto con reborde anterosuperior apenas interrumpido medialmente, ángulos posterolaterales del propódeo angulados, formando una carena, segundo esterno del metasoma sin protuberancias y fuertemente angulado.

Descripción. Holotipo hembra. Coloración (Fig. 3H): negra, con las siguientes partes marfil: línea angosta superior en la órbita externa; banda apical delgada y solo presente en la parte central del pronoto; bandas apicales en primer y segundo tergo. Son ferrugíneo: antenas; pequeña mancha en la frente entre la base de las antenas; clípeo; mandíbulas en sus bordes externo e interno y en su ápice ferrugíneo oscuro; tégulas; patas a partir de menos de la mitad de los fémures. Alas en gran parte ferrugíneas, solo con el ápice en parte infumado. Cabeza (Figs. 2C-2D): más ancha que larga (ancho $\bar{X}: 3,9$ mm; largo $\overline{\mathrm{X}}$ : 3,3 mm); distancia interorbital máxima mayor que el largo del ojo (1:0,7); órbitas internas profundamente escotadas en su mitad superior, de modo que la distancia entre las escotaduras es casi el doble de la distancia interorbital media (1:0,5); clípeo basalmente proyectado hacia adelante en vista lateral, más ancho que largo (1:0,9), apicalmente emarginado, sin reborde translúcido, y con dos dientes apicales redondeados, bien 
representados y levemente proyectados lateralmente; mandíbulas largas, con 4 dientes (considerando como un diente el ápice de la mandíbula), cara dorsal provista de carenas longitudinales y cara ventral cóncava; antenas: escapo, más de tres veces más largo que ancho $(1: 0,2)$. Mesosoma (Figs. 2A-2B): con apariencia lustrosa, más largo que ancho $(1: 0,8)$; pronoto con reborde anterosuperior apenas interrumpido en su parte media; en vista dorsal ángulos pronotales formando un ángulo obtuso; propódeo con los ángulos posterolaterales carenados y la superficie lateral en gran parte brillante, con rugosidades. Metasoma (Figs. 2A-2B): en el primer tergo, la cara anterior pasa a la dorsal formando un ángulo fuerte con un surco longitudinal medio que da origen a una hendidura basal; el segundo esterno es redondeado basalmente en vista lateral. Puntuación: cabeza con puntos marcados, en el vertex de menor diámetro que el espacio entre ellos, hacia el clípeo y la escotadura de los ojos tiene un diámetro mayor que la distancia entre ellos; mesosoma con puntos marcados, mayoritariamente con un diámetro menor que el espacio entre ellos; clípeo con algunos puntos de un diámetro menor que el espacio entre ellos, en el ápice más marcados y de mayor tamaño que en su zona media y basal; el primer tergo del metasoma con puntuación marcada que presenta un diámetro menor que el espacios entre ellos; en los demás tergos no se aprecia puntuación. Pilosidad: corta, hirsuta, espaciada entre ella, negra sobre las zonas de tegumento negro y marfileña en las zonas de tegumento de ese color, no apreciable a simple vista.

Macho. Desconocido.

Material examinado. Holotipo hembra de: Chile, Región de Atacama, Provincia Copiapó, cordillera de Copiapó, 27²43' S, 6941' O, 15-enero-2011, leg. T. Fichet (MNHN). Paratipos: Chile, Región de Coquimbo, Provincia del Elqui, Punta de Choros, 28-octubre-2009, 1 , leg. C. Fortino (CRBM); Región Metropolitana de Santiago, Provincia Cordillera, Lagunillas km 5,5, 18-diciembre-2005, 1, leg. R. Barrera M. (MNHN).

Etimología. Especie dedicada a Thomas Fichet Lagos, gran amigo y entusiasta de la entomología, quien nos ha proporcionado uno de los pocos ejemplares conocidos de esta especie.

Distribución. Chile, entre las regiones de Atacama y Metropolitana de Santiago, tramo que corresponde a las provincias biográficas de Atacama, Coquimbo y Santiago (Morrone 2015).

Hypodynerus bruchii (Brèthes, 1903)

Odynerus bruchii Brèthes, 1903

Hypodynerus bruchi [!]; Willink y Chiappa (1993)

(Figs. 3B-3C)

Material examinado. Chile, Región de La Araucanía, Provincia Malleco, Nahuelbuta, 1200

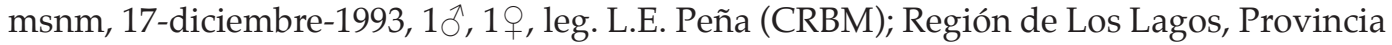
Llanquihue, Lago Chapo, enero-1969, 1§̋, leg. L. Peña (MNHN); 12-febrero-1971, 1q, leg. M. Pino (MNHN); Región de Aysén del General Carlos Ibáñez del Campo, Provincia de Capitán Prat, Puerto Cristal, 21-enero-1956, 1ㅇ, (MNHN).

Distribución. Argentina, Patagonia, Volcán Tronador cerca de la ciudad de Bariloche (Brèthes 1903), correspondiente a la Provincia biogeográfica de la Patagonia (Morrone 2015). Chile, entre las regiones de La Araucanía y Aysén del General Carlos Ibáñez del Campo, tramo correspondiente a las provincias biográficas del Maule y del bosque valdiviano (Morrone 2015). 


\section{2}

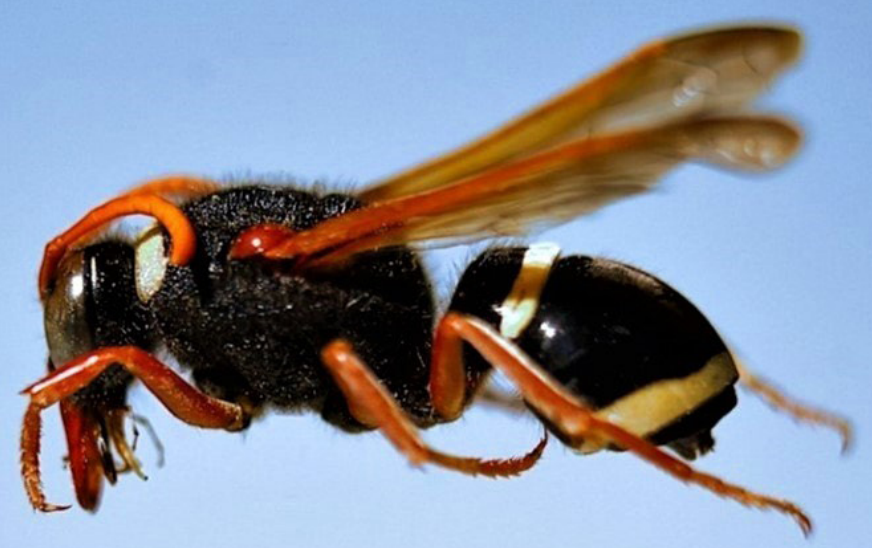

\section{$10 \mathrm{~mm}$}

A

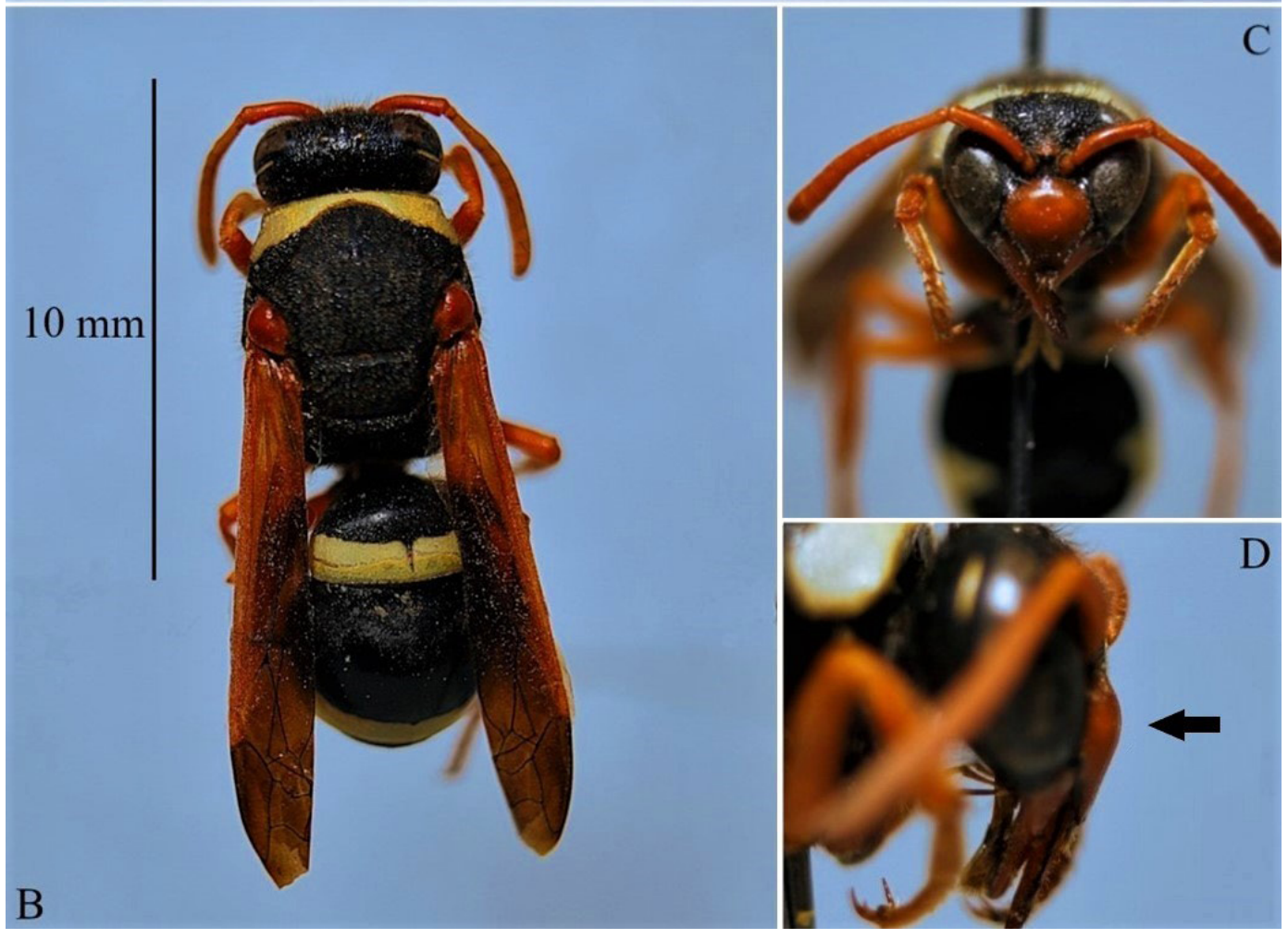

Figura 2. Hypodynerus ficheti sp. nov. A. Vista lateral izquierda. B. Vista dorsal. C. Cabeza vista frontal. D. Clípeo vista lateral. / A. Left side view. B. Dorsal view. C. Head frontal view. D. Clypeus lateral view. 


\section{Hypodynerus caupolicanus (Reed, 1893) \\ Odynerus caupolicanus Reed, 1893 \\ = Odynerus sotoi Reed, 1893}

Hypodynerus caupolicanus; Bequaert y Ruiz (1943)

(Figs. 3D-3E)

Material examinado. Chile, Región Coquimbo, Provincia del Elqui, Vicuña, 11-noviembre-2008, 1ㅇ, leg. G. Castillo (CRBM); Región Metropolitana de Santiago, Quilicura, 24-septiembre-1989, 1ㅇ, leg. G. Aceituno (CRBM); Lo Aguirre, 2ㅇ, leg. R. Barrera M. (CRBM); Provincia de Santiago, Las Condes, 20-diciembre-1970, 1이, leg. T. Ramírez (MNHN).

Distribución. Chile, entre las regiones de Atacama y Libertador General Bernardo O'Higgins, tramo correspondiente a las provincias biogeográficas de Atacama, Coquimbo y Santiago, respectivamente (Morrone 2015).

Hypodynerus cerberus Bequaert y Ruiz, 1943

Hypodynerus caupolicanus cerberus Bequaert y Ruiz, 1943

Hypodynerus cerberus; Willink y Chiappa (1993)

(Figs. 3F-3G)

Material examinado. Chile, Región del Maule, Provincia de Talca, Vilches Alto camino a piedras blancas, 13 al 16-enero-2018, 1ㅇ, leg. R. Barrera M. (CRBM); Región de Ñuble, Provincia de Diguillín, Shangri-La, 21-enero-2011, 1이, leg. P. Pinto (CRBM).

Distribución. Chile, entre las regiones de Valparaíso y Ñuble, tramo correspondiente a las provincias biogeográficas de Santiago y el Maule, respectivamente (Morrone 2015).

Hypodynerus herbsti Bequaert y Ruiz, 1943

Hypodynerus caupolicanus herbsti Bequaert y Ruiz, 1943

Hypodynerus herbsti; Willink y Chiappa (1993)

(Figs. 3I-3J)

Material examinado. Chile, Región de Coquimbo, Provincia del Elqui, Coquimbo, 18/20-Octubre-1990, 1의, leg. L. Peña (CRBM); Vicuña, 18/20-Octubre-1990, 1이, leg. L. Peña (CRBM); Región Metropolitana de Santiago, Provincia Cordillera, El Alfalfal, 27-octubre-2007, 3q, leg. R. Barrera M. (CRBM); 4-noviembre-2007, 1ㅇ, leg. R. Barrera M. (CRBM); camino El Alfalfal, 16-noviembre-2013, 4우, leg. R. Barrera M. (CRBM); El Manzano, 17-noviembre-2018, 1\%; Región de La Araucanía, Provincia de Malleco, Malalcahuello, camino a la Sierra Nevada, 12 al 18-enero-2008, 2o, leg. R. Barrera M. (CRBM).

Distribución. Chile, entre las regiones de Coquimbo y La Araucanía, tramo correspondiente a las provincias biogeográficas de Coquimbo, Santiago y el Maule (Morrone 2015).

\section{Clave para hembras de las especies del grupo de Hypodynerus caupolicanus}

1. Postescudete ferrugíneo o por lo menos con rastros de ferrugíneo, clípeo negro con el ápice ferrugíneo (pudiendo ser completamente negro) (Fig. 3B). Zonas sur y austral de Chile y Patagonia Argentina H. bruchii Brèthes 
- Postescudete sin rastros de ferrugíneo (pudiendo ser marfileño o negro) ....................2

2. Con carenas en los ángulos posterolaterales del propódeo..............................

- Sin carenas en los ángulos posterolaterales del propódeo ................................10

3. Clípeo basalmente proyectado hacia adelante en vista lateral (Fig. 2D), formas robustas ..

- Clípeo convexo en vista lateral (Fig. 1D), no proyectado basalmente en vista lateral.......4

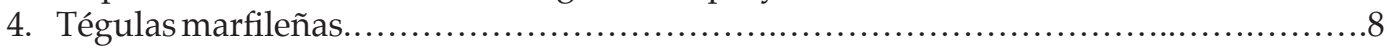

- Tégulas ferrugíneas.................................................................. 5

5. Clípeo mayormente marfileño con bordes negros, en algunos casos formado una amplia mancha central marfileña, apicalmente puede ser ferrugíneo........................................7

- Clípeo sin diseños marfileños (mayormente negro o ferrugíneo)..............................6

6. Antenas, tégulas y fémures totalmente ferrugíneos, banda en el pronoto en algunos casos presente o disminuida. Chile, zonas centro y sur (Fig. 3F).

H. cerberus Bequaert y Ruiz

7. Postescudete con diseños marfil y bandas apicales marfileñas en el primer y segundo tergo. Chile, zonas norte chico, centro y sur (Fig. 3I). .H. herbsti Bequaert y Ruiz

8. Clípeo marfileño, apicalmente puede ser ferrugíneo, postescudete con diseños marfil y bandas apicales marfileñas desde el primer hasta el sexto tergo. Chile, zonas norte chico y centro (Fig. 3D) ............................................. caupolicanus Reed

9. Clípeo apicalmente emarginado, con dos dientes apicales bien desarrollados y levemente proyectados lateralmente (Figs. 2A-2E). Chile, zonas norte chico y centro....................................................................................... H. ficheti sp. nov.

10. Clípeo mayormente ferrugíneo con bordes negros, en algunos casos formado una amplia mancha central ferrugínea, banda en el pronoto, mancha dorsal en las mesopleuras, postescudete y bandas apicales en el primer y segundo tergo marfileñas (mancha dorsal en las mesopleuras y postescudete marfileño pueden no estar presentes), tégulas ferrugíneas. Chile, zonas norte chico y centro (Figs. 1A-1E).

\section{Lista actualizada y esquemas de color típicos de las especies del grupo de Hypodynerus caupolicanus}

Hypodynerus aceitunoi sp. nov. (Fig. 3A)

Distribución. Chile, zonas norte chico y centro (endémica)

Hypodynerus bruchii (Brèthes, 1903) (Figs. 3B-3C)

Distribución. Argentina y Chile, zonas sur y austral (Brèthes 1903; Willink y Chiappa 1993)

Hypodynerus caupolicanus (Reed, 1893) (Figs. 3D-3E)

Distribución. Chile, zonas norte chico y centro (endémica) (Willink y Chiappa 1993)

Hypodynerus cerberus Bequaert y Ruiz, 1943 (Figs. 3F-3G)

Distribución. Chile, zonas centro y sur (endémica) (Willink y Chiappa 1993)

Hypodynerus ficheti sp. nov. (Fig. 3H)

Distribución. Chile, zonas norte chico y centro (endémica)

Hypodynerus herbsti Bequaert y Ruiz, 1943 (Figs. 3I-3J)

Distribución. Chile, zonas norte chico, centro y sur (endémica) (Willink y Chiappa 1993) 
3

A
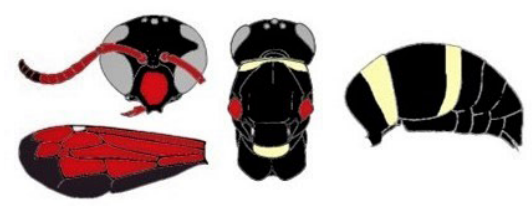

B
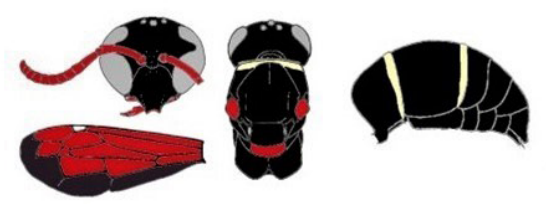

C

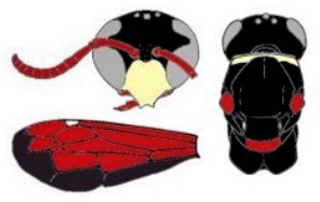

D
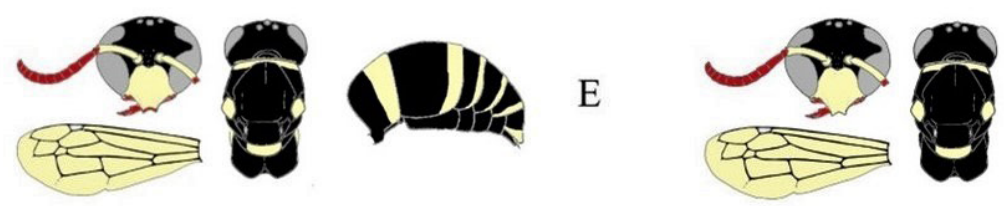

F
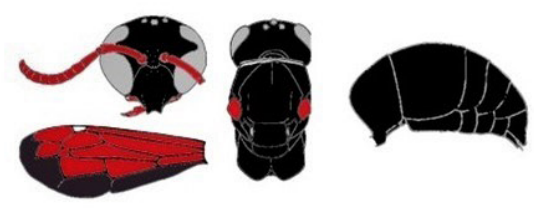

G
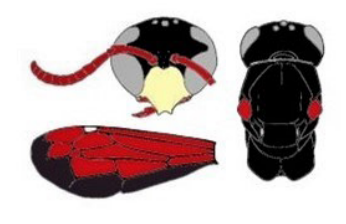

H
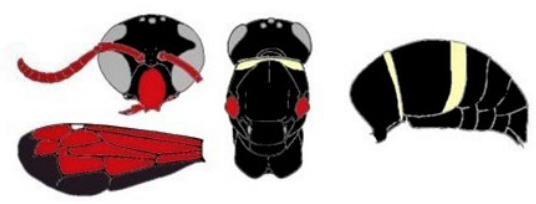

E
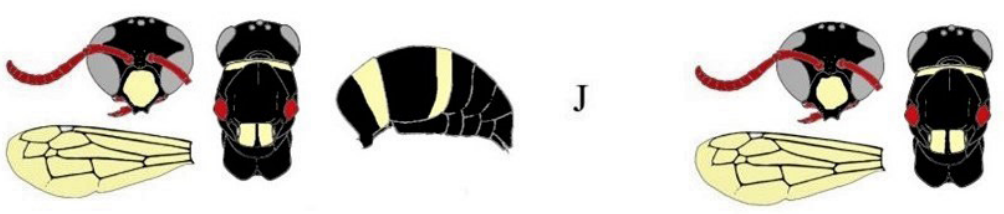

* Los esquemas son solo referenciales no representan la morfología de cada especie, solo se representa la coloración típica.

Figura 3. Esquemas de coloración corporal. A. Hembra de Hypodynerus aceitunoi sp. nov. B-C. Hembra y macho de Hypodynerus bruchii (Brèthes). D-E. Hembra y macho de Hypodynerus caupolicanus (Reed). F-G. Hembra y macho de Hypodynerus cerberus Bequaert y Ruiz. H. Hembra de Hypodynerus ficheti sp. nov. I-J. Hembra y macho de Hypodynerus herbsti Bequaert y Ruiz. / Body coloring schemes. A. Female Hypodynerus aceitunoi nov. sp. B-C. Female and male Hypodynerus bruchii (Brèthes). D-E. Female and male Hypodynerus caupolicanus (Reed). F-G. Female and male Hypodynerus cerberus Bequaert \& Ruiz. H. Female Hypodynerus ficheti nov. sp. I-J. Female and male Hypodynerus herbsti Bequaert \& Ruiz. 


\section{Discusión}

En Chile los representantes del género Hypodynerus presenta variabilidad en su coloración, en algunas especies los diseños marfileños y ferrugíneos pueden no estar presentes; esta situación se ha reportado en ejemplares de las zonas meridionales y de altura (Barrera-Medina 2011); sin embargo, el color de las alas no presenta cambios, aparentemente corresponde a un carácter constante que debería ser considerado en la identificación de las especies y en la descripción de nuevos taxa.

Hypodynerus aceitunoi, $H$. bruchii e $H$. ficheti no presentan protuberancia mediana en el segundo esternito; la primera de ellas tiene los ángulos posterolaterales del propodeo redondeados y las tres últimas especies presentan carenas en los ángulos posterolaterales del propodeo, además, todas presentan lamela pronotal completa o apenas interrumpida, por lo que se proponen como especies pertenecientes al grupo de H. caupolicanus.

El caso de $H$. caupolicanus, $H$. cerberus y $H$. herbsti, todas ellas presentan lamela pronotal apenas interrumpida medialmente, además de carenas en los ángulos posterolaterales del propodeo, no presentan protuberancia mediana en el segundo esternito y solo se presentan diferencias de coloración según lo indicado por Bequaert y Ruiz (1943), por lo que posiblemente sean diferentes formas de una misma especie, especialmente $H$. caupolicanus y $H$. herbsti que muestran cercanía en la coloración (con abundantes diseños marfileños) y comparten el mismo color de alas, es $H$. cerberus quien se aleja de la coloración típica no presentando los diseños marfileños característicos de las especies antes mencionadas. Bequaert y Ruiz (1943) indican que el color del clípeo de la hembra es negro con la excepción de un ejemplar de Concepción que tiene mayormente ferrugíneo el clípeo, esta observación fue considerada en la clave propuesta, quedando pendiente revisar en profundidad estas especies.

Con respecto a $H$. antuco, Bequaert y Ruiz (1941) indican que es una sinonimia de H. labiatus (Haliday), Willink (1981) la compara con especies del grupo de H. labiatus revalidándola como buena especie e indicando que pertenece al grupo de $H$. caupolicanus, además menciona que el tubérculo del segundo esternito es muy poco evidente. Según esta aseveración, podemos indicar que dicho carácter está presente, pero de forma muy discreta; de este modo, se propone que esta especie debe pertenecer al grupo de H. labiatus debido a que presenta el pronoto sin reborde o lámina anterosuperior, esterno II fuertemente angulado en la base y con una protuberancia media más o menos definida.

Los integrantes del grupo de $H$. caupolicanus se distribuyen desde la Región de Atacama hasta la Región de Aysén y aparentemente no lograron superar el desierto de Atacama hacia el norte. A nivel sudamericano, el grupo queda conformado por seis especies. Con los nuevos taxones aquí descritos, en Chile el género Hypodynerus agrupa a treinta y dos especies, las que se distribuyen ampliamente de mar a cordillera en gran parte del territorio chileno, con excepción de la Antártida.

\section{Agradecimientos}

Alfredo Ugarte, Carlos Vidal, Cesar Fortino, Guido Castillo, Guillermo Aceituno, Hirohiko Nagase (material enviado desde Japón), Juan Carlos González, Pablo Pinto, Richard Honour y Thomas Fichet por ceder material para estudio. Agradecemos a los curadores Mario Elgueta y Francisco Urra del Museo Nacional de Historia Natural de Chile por permitirnos revisar la colección de Hymenoptera y por la lectura crítica del escrito hecha por F. Urra. Apreciamos las importantes correcciones y sugerencias realizadas por los revisores anónimos. 


\section{Literatura Citada}

Barrera-Medina, R. (2011) Descripción de una nueva avispa alfarera del norte chico chileno, Hypodynerus anae n. sp. (Hymenoptera: Vespidae: Eumeninae). Boletín de la Sociedad Entomológica Aragonesa, 48: 157-162.

Barrera-Medina, R. y Garcete-Barrett, B.R. (2016) Primer reporte de Hypodynerus rufinodis (du Buysson, 1912) (Hymenoptera: Vespidae: Eumeninae) en el extremo Norte de Chile. Boletín del Museo Nacional de Historia Natural del Paraguay, 20(2):148-153.

Bequaert, J. y Ruiz, F. (1943) A revision of the Vespidae (Hymenoptera, Diploptera) of Chile. Part II. Subfamily Eumeninae. Genus Hypodynerus. Revista Chilena de Historia Natural, 45: 69-103. [1941]

Brèthes, J. (1903) Los euménidos de las repúblicas del Plata. Anales del Museo Nacional de Buenos Aires (serie 3), 2: 231-320.

Carpenter, J.M. y Garcete-Barrett, B.R. (2003) A key to the Neotropical genera of Eumeninae (Hymenoptera: Vespidae). Boletín del Museo Nacional de Historia Natural del Paraguay, 14(12): 52-73. [2002]

Morrone, J.J. (2015) Biogeographical regionalisation of the Andean region. Zootaxa, 3936: 207-236.

Reed, E.C. (1893) Entomolojía chilena. Sinópsis de las avispas chilenas pertenecientes a la familia Odyneridae. Anales de la Universidad de Chile, 84: 873-897.

Willink, A. (1970) Revisión del género Hypodynerus Saussure (Hym., Eumenidae). I Grupo de Hypodynerus humeralis (Hal.). Acta Zoológica Lilloana, 25(20): 229-278.

Willink, A. (1978a) Revisión del género Hypodynerus (Hym., Eumenidae) II. Grupo de $H$. tuberculiventris. Acta Zoológica Lilloana, 33(1): 5-13.

Willink, A. (1978b) Revisión del género Hypodynerus (Hymenoptera, Eumenidae) III. Grupo de H. excipiendus. Acta Zoológica Lilloana, 33(1): 15-31.

Willink, A. (1981) Una nueva especie de Hypodynerus y observaciones sinonímicas sobre otras (Hym. Eumenidae). Revista Peruana de Entomología, 24(1): 55-58.

Willink, A. y Chiappa, E. (1993) Lista de las especies chilenas de la familia Vespidae (Hymenoptera: Vespidae). Acta Entomológica Chilena, 18: 119-125.

Zavattari, E. (1912) Materialien für une Monographie der Neotropischen Eumeniden. Archiv für Naturgeschichte, 78 A(4): 1-272 + 2 pl. 